\title{
Diamagnetic Length Scales of Condon Domain Phase in Lifschitz-Kosevich-Shoenberg Approximation
}

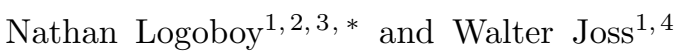 \\ ${ }^{1}$ Grenoble High Magnetic Field Laboratory, MPI-FKF and CNRS P.O. 166X, F-38042 Grenoble Cedex 9, France \\ ${ }^{2}$ The Racah Institute of Physics, The Hebrew University of Jerusalem, 91904 Jerusalem, Israel \\ ${ }^{3}$ Institute of Superconductivity, Department of Physics, Bar-Ilan University, Ramat-Gan 52900, Israel \\ ${ }^{4}$ Université Joseph Fourier, B.P. 53, F-38041 Grenoble Cedex 9, France
}

(Dated: November 21, 2018)

\begin{abstract}
Equilibrium properties of non-uniform diamagnetic phase in normal metals (Condon domains) are studied theoretically in the framework of Lifschitz-Kosevich-Shoenberg (LKS) approximation. It is found that characteristic diamagnetic lengths of the phase, e. g. a period of domain structure and width of interface boundary between domains, as well as specific surface energy of domain wall, are strongly affected by electron correlations and depend on temperature, magnetic field and purity of the sample. The developed theory is in a good agreement with existent experiment data.
\end{abstract}

PACS numbers: 71.18+y; 75.30.Kz; 75.60.Ch; 75.70.Kw

Keywords: D. Condon domains; D. Diamagnetic phase transition; D. dHvA effect

\section{INTRODUCTION}

Diamagnetic instability of electron gas in normal metals under quantizing magnetic field and low temperature is a result of strong electron correlations induced by magnetic field. It gives rise to a phase transition with formation of complex domain patterns [1]-[3]. The phase transition can occur at every period of dHvA oscillations and is handled by the tools of catastrophe theory [4]. The symmetric pitchfork bifurcation gives rise to the secondorder phase transition on temperature at the center of the dHvA period, while the deviation from the center results in a phase transition of the first order both in temperature and magnetic field. The diamagnetic phase transition has received recently much attention due to a number of unusual phenomena for the physics of diamagnetism, e. g. formation of complex branch structures [3], [5], strong dependence of magnetic phase diagrams on Fermi-surface topology [6], [7], presence of diamagnetic hysteresis in magnetization curves [8], existence of persistent currents [9] which results in a discontinuity of magnetic induction along the interface boundaries of regular domain patterns [2], 5].

The stratification of the sample into the laminar domain structure, or Condon domains (CDs), was first observed in a plate-like sample of silver [2] by measuring the NMR frequency splitting due to a presence of two different kinds of domains. Later, the increase in absorption of the low-frequency electromagnetic field (helicons) in aluminum under cooling below critical temperature was explained by the onset of the diamagnetic phase transition [10]. Due to the technical difficulties of the experimental observation of CDs in normal metals, these results have remained the only references on the phenomenon of diamagnetic instability. Recently, the existence of CDs

*Electronic address: logoboy@phys.huji.ac.il was confirmed by methods of muon spin-rotation spectroscopy in beryllium, white tin, lead, aluminum and indium [11], [12]. The formation of rather complicated diamagnetic structure in silver was demonstrated by use of a set micro Hall probes [5]. Further development of the experimental technique including the standard ac method with different modulation levels, frequencies and magnetic field ramp rates allowed us to reconstruct the magnetization reversal in beryllium [8]. The detection of giant nonlinear response at the crossing critical point, $a \rightarrow 1$, where $a=\mu_{0} \max \{\partial M / \partial B\}$ is a differential magnetic susceptibility, offered a way to construct the diamagnetic phase diagrams [5], 13]-[15].

Despite numerous experimental evidence for diamagnetic instability in normal metals, there remain some open fundamental questions related to the diamagnetic length scales of the CD phase. The important information about the size of the domains, the domain wall (DW) width and surface energy of the interface boundaries is still lacking. The attempt of direct measurement of the period of the domain structure in plate-like sample of silver by the Hall probe technique [5] revealed the value of $\sim 150 \mu \mathrm{m}$ instead of expected one $\sim 30 \mu \mathrm{m}$ at the conditions of experiment (applied magnetic field $\mu_{0} H=10$ $\mathrm{T}$, temperature $T=1.3 \mathrm{~K}$ and plate thickness $L \approx 1$ $\mathrm{mm})$. Calculation of the contribution of the short-range interaction (on the scale of cyclotron radius $r_{c}$ ) into the free energy density was carried out by Privorotskii [16] Unfortunately, the investigation of DW width, specific surface energy of interface boundary and the size of the domains in [16] is restricted by a limit case $a \rightarrow 1$. The direct applicability of the theory of domain structures developed in physics of magnetic materials [17] remains under question. So far there is no reliable theory of the temperature and magnetic field dependence of the diamagnetic length scales in a full range of existence of the non-uniform phase.

Motivated by these problems, we present the systematic theoretical studies of the diamagnetic length scales 
for normal metals in LKS approximation. Our investigation is based on the diamagnetic phase diagrams in a full range of temperature and magnetic field. We derive equations which allow us to evaluate the DW width $\delta$, DW-specific surface energy $\sigma$ and the period of the domain structure $D$ by use of experimentally measured parameter, e. g. the value of a jump of magnetic induction at the interface boundaries. We calculate the temperature and magnetic field dependence of $\delta, \sigma$ and $D$ and study the influence of the impurity of the sample on these characteristics. We show that for a plate-like sample of silver with thickness $L \sim 1 \mathrm{~mm}$ a typical value of the period of the laminar diamagnetic structure is $D \sim 0.1$ $\mathrm{mm}$ which is in two orders of value higher than the average period of the ferromagnetic domain structure for the sample of iron of the same shape. Deep inside the diamagnetic phase, the DW width $\delta$ is almost constant, falling into interval $\sim 1-2 \mu \mathrm{m}$ which is of the same order of value as the width of interface boundary between domains in low-anisotropy magnetic materials such as thin magnetic films of permalloy $\left(\mathrm{Ni}_{80} \mathrm{Fe}_{20}\right.$ [17]). Close to the critical point when $\left(r_{c} / L\right)^{2 / 3} \ll \alpha \ll 1(\alpha=a-1$ is increment of differential magnetic susceptibility $a$ relative to the critical value 1 ), the DW width $\delta$ is scaled as a coherence length which defines the range of correlations, and diverges with the critical index $\nu=1 / 2$ in accordance with mean-field theory. Approaching at the critical point, a period of the domain structure decreases, and the domain structure becomes more dense, as a result of the essential decrease in positive energy of the interface boundaries. In the nearest vicinity of the critical point when $\alpha \lesssim\left(r_{c} / L\right)^{2 / 3}$, the stripe domains with welldefined DWs transform into modulated domain structure (see, also [3] ).

The paper is organized as follows. In Sec. III, we introduce the model and basic equations. In Sec. III we calculate the temperature and magnetic field dependences of DW width, specific surface energy of the DW and period of the domain structure and discuss the influence of impurities on these characteristics. Finally, in Sec. IV] we summarize the conclusions.

\section{MODEL}

In a single-harmonic approximation the properties of correlated electrons in normal metals under the conditions of the strong dHvA effect are described by the free energy functional [1]

$$
G(y ; a, x)=a \cos (x+y)+\frac{1}{2} y^{2}+\frac{1}{2} a r_{c}^{2}\left(\partial_{\zeta} y\right)^{2},
$$

where the small-scale magnetic field $x=k \mu_{0}\left(H-H_{a}\right)$ is the increment of the large-scale internal magnetic field $\mu_{0} H$ and the applied magnetic field $\mu_{0} H_{a}, y=$ $4 \pi k M$ is oscillating part of reduced magnetization, $k=$ $2 \pi F /\left(\mu_{0} H_{a}\right)^{2}=2 \pi / \Delta H, F$ is the fundamental frequency of the $\mathrm{dHvA}$ oscillations corresponding to the extremal cross-section of the Fermi surface, $\Delta H$ is the dHvA period and $a=\mu_{0} \max \{\partial M / \partial B\}$ is the differential magnetic susceptibility [1]. In physical units $x$ is of the order of $\sim 1-10 \mathrm{mT}$ depending on the properties of the electron system, while $\mu_{0} H$ is $\sim 1-10 \mathrm{~T}$. The gradient term in Eq. (11) is the lowest-order term in a full gradient expansion [16] which accounts for the short-range correlations on the scale of $r_{c}$ ( $\zeta$ is coordinate).

In the case of the ellipsoidal Fermi surface, the temperature and magnetic field dependence of the reduced amplitude of dHvA oscillations $a$ is defined by [1]

$$
a=a_{0}\left(\mu_{0} H\right) \frac{\lambda\left(\mu_{0} H, T\right)}{\sinh \lambda\left(\mu_{0} H, T\right)} \exp \left[-\lambda\left(\mu_{0} H, T_{D}\right)\right],
$$

where $\lambda\left(\mu_{0} H, T\right)=2 \pi^{2} k_{B} T / \hbar \omega_{c}, k_{B}$ is the Boltzmann constant, $\hbar$ is the Planck constant, $\omega_{c}=\left(e / m_{c}\right) \mu_{0} H$ is cyclotron frequency, $e$ is absolute value of the electron charge, $m_{c}$ is the cyclotron mass, and $T_{D}=\hbar / 2 \pi k_{B} \tau$ is the Dingle temperature inversely proportional to the scattering lifetime $\tau$ of conduction electrons. The limiting amplitude $a_{0}=\left(H_{m} / H\right)^{3 / 2}$ in Eq. (2) is the combination of temperature-independent factors [1], and $\mu_{0} H_{m}=$ $\left(10.4 \eta \epsilon_{F}^{2}\right)^{2 / 3}$ is the maximal magnetic field above which diamagnetic phase transition does not occur at any temperature, $\epsilon_{F}$ is Fermi energy in $e V, \eta=m_{c} / m$ and $m$ electron mass. The validity of Eq. (2) is restricted by the application to the spherical (or almost spherical) Fermi surface sheets, which is the case of noble metals [1]. Equation $a\left(\mu_{0} H, T, T_{D}\right)=1$ defines the locus of critical points, e. g. a surface in three dimensions $\mu_{0} H-T-T_{D}$ which separates the uniform and CD phases. The calculated phase diagrams are in a good agreement with the experimental data on measurement of amplitude of the third harmonic of the $a c$ susceptibility [13], justifying the applicability of $a$ Eq. (2) for belly oscillations in silver.

Minimization of the free energy $G$ Eq. (1) with respect to magnetization $y$ at the center of dHvA period, $x=0$, leads to differential equation $a \sin y-y+a r_{c}^{2} \partial_{\zeta \zeta}^{2} y=0$ which can be integrated

$$
\int_{y_{0}}^{y} \frac{d y}{f(y ; a)}=\frac{\zeta}{a^{1 / 2} r_{c}}
$$

where $f(y ; a)=\left(y^{2}+2 a \cos y-C\right)^{1 / 2}$. Eq. (3) forms the basis for investigation of non-uniform phases in onedimensional problems. A proper choice of integration constant $C$ is dictated by specific boundary conditions. We assume the existence of periodic domain structure with alternative magnetization $\pm y_{0}$ in neighboring domains, defined in explicit form by equation $y_{0}=a \sin y_{0}$ (see, Fig. 1). The period of the domain structure $D$ is defined by competition between the long-range dipoledipole interaction dependent on the size and the shape of the sample, and short-range electron interaction on the scale of $r_{c}$ which gives rise to positive energy of interface boundaries. Inserting $C=y_{0}^{2}+2 a \cos y_{0}$ into Eq. (3), we calculate the equilibrium structure of DW with the 

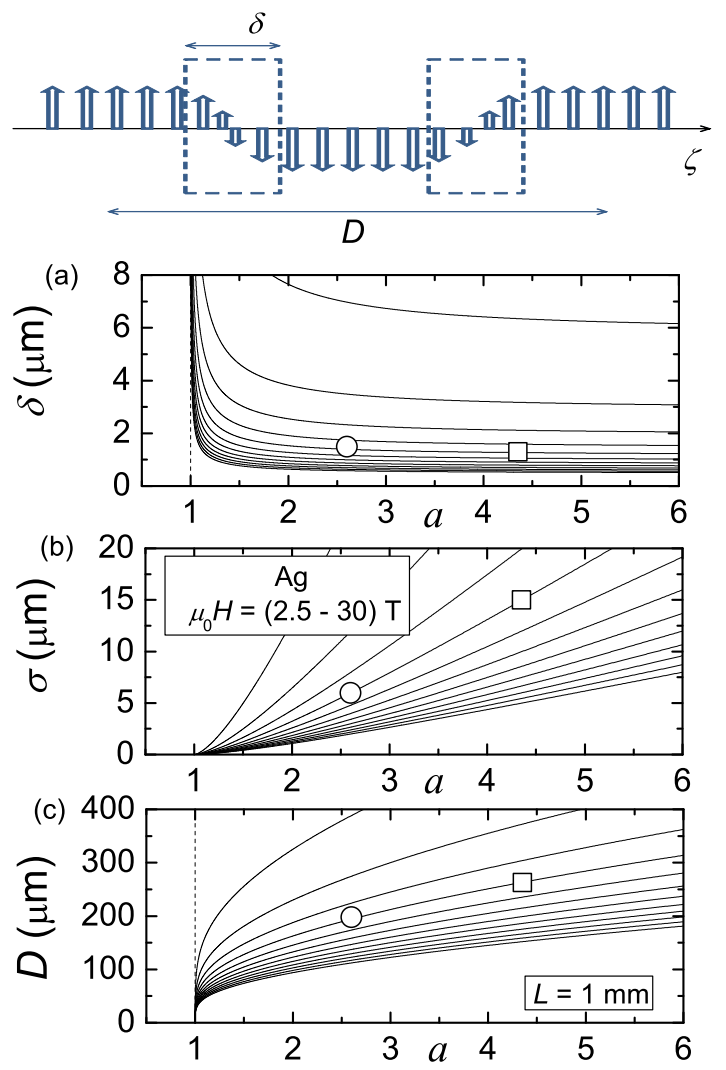

FIG. 1: (color online). The upper panel shows a setup of the system. (a) DW width $\delta=\delta(a)$, (b) specific surface energy $\sigma=\sigma(a)$, and (c) period of the domain structure $D=D(a)$ are plotted as functions of differential magnetic susceptibility $a$ at different values of the magnetic field $\mu_{0} H$. The magnetic field increases in steps of $2.5 \mathrm{~T}$ starting from $2.5 \mathrm{~T}$ (from top to bottom) which corresponds to decreasing values of $r_{c}=2.91,1.45,0.97,0.73,0.58,0.48,0.42,0.36,0.32$, $0.29,0.26$ and $0.24 \mu \mathrm{m}$. Close to the point $a \rightarrow 1+0^{+}$all characteristics show critical behavior. The horizontal asymptote for $\delta$ is $2 r_{c}$ (not shown). Circle (square) corresponds to the values of characteristic lengths calculated at the conditions of experiment 2] (5]). The nearest vicinity of the critical point $\left(\alpha \lesssim\left(r_{c} / L\right)^{2 / 3}\right)$ where periodic domain structure transforms into the modulated structure is excluded from the consideration.

following characteristic DW width, $\delta=\delta(a)$, and specific surface energy of DW, $\sigma=\sigma(a)$ (see, e. g. 18])

$$
\begin{gathered}
\delta=2 a^{1 / 2} r_{c} \lim _{\epsilon \rightarrow 0} \frac{1}{\ln \epsilon^{-1}} \int_{0}^{(1-2 \epsilon) y_{0}} \frac{d y}{f(y ; a)} \\
\sigma=2 a^{1 / 2} r_{c} \int_{0}^{y_{0}} d y f(y ; a) .
\end{gathered}
$$

For a plate-like sample of thickness $L$, the standard procedure of minimization of total free energy of the periodic domain structure with period $D$, containing two terms, e. g. dipole-dipole energy $\left(7 / \pi^{3}\right) \zeta(3) y_{0}^{2} D$, where $\zeta(3)$ is zeta-function, and surface energy of separation of two domains $(2 L / D) \sigma$ [19], allows us to calculate a

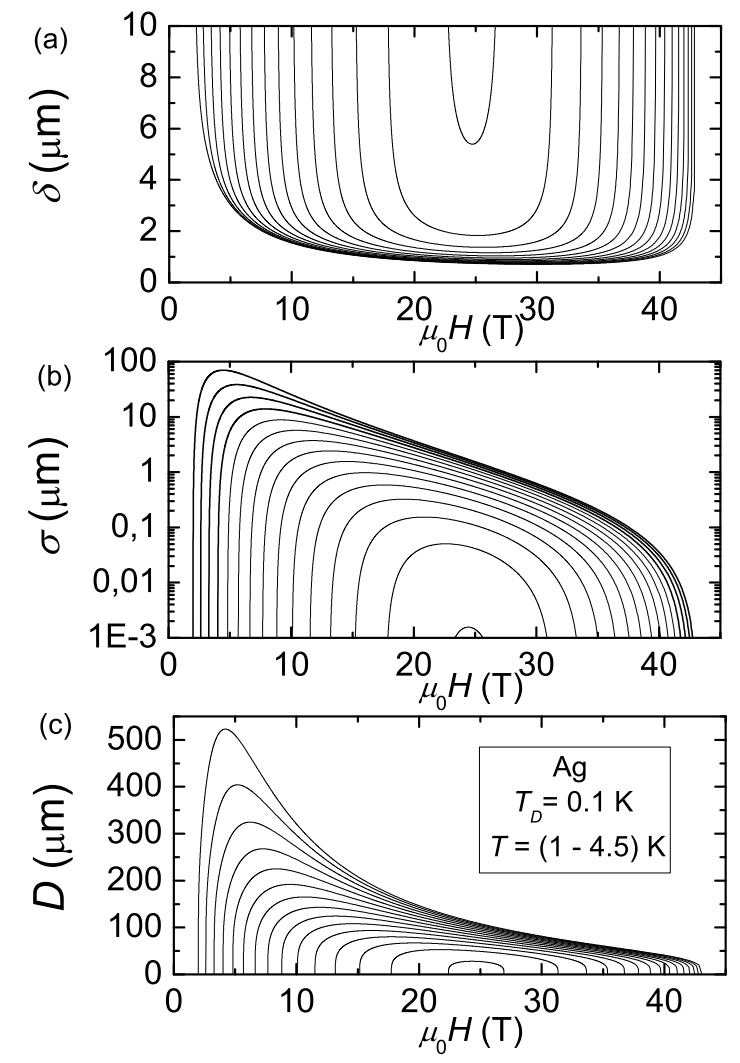

FIG. 2: (a) Magnetic field dependence of DW width $\delta=$ $\delta\left(\mu_{0} H\right)$, (b) specific surface energy $\sigma=\sigma\left(\mu_{0} H\right)$, and (c) period of the domain structure $D=D\left(\mu_{0} H\right)$ at Dingle temperature $T_{D}=0.1 \mathrm{~K}$ and different temperatures $T$. The temperature increases in steps of $0.25 \mathrm{~K}$ starting from $1 \mathrm{~K}$ (from bottom to top in (a) and from top to bottom in (b) and (c)).

period of the domain structure

$$
D=\frac{(2 \pi)^{3 / 2}}{[7 \zeta(3)]^{1 / 2}} \frac{(\sigma L)^{1 / 2}}{y_{0}}
$$

The DW width $\delta$ Eq. (4), specific surface energy of DW $\sigma$ Eq. (5) (material-dependent length) and period of the domain structure $D$ Eq. (6) form a complete set of characteristic diamagnetic sizes for CD phase. In particular, the dimensionless characteristic length $\sigma / 2 L$ (also referred as material constant) plays an important role in studies of evolution of the domain structures [20]. The existence of the well-defined domain structure implies $\delta \ll D / 2$.

\section{RESULTS AND DISCUSSIONS}

Close to the critical point when $\alpha \ll 1$ Eqs. (3)-(6) can be simplified by using the expansion of trigonometric functions in powers of $y \leq y_{0}=(6 \alpha)^{1 / 2} \ll 1$. In this case, the DW structure is described by the function $y=$ $y_{0} \tanh \left(\alpha^{1 / 2} \zeta / r_{c}\right)$ 3] with following asymptotic behavior of the characteristic lengths $\delta$ Eq. (4), Eq. (5) and $D$ 

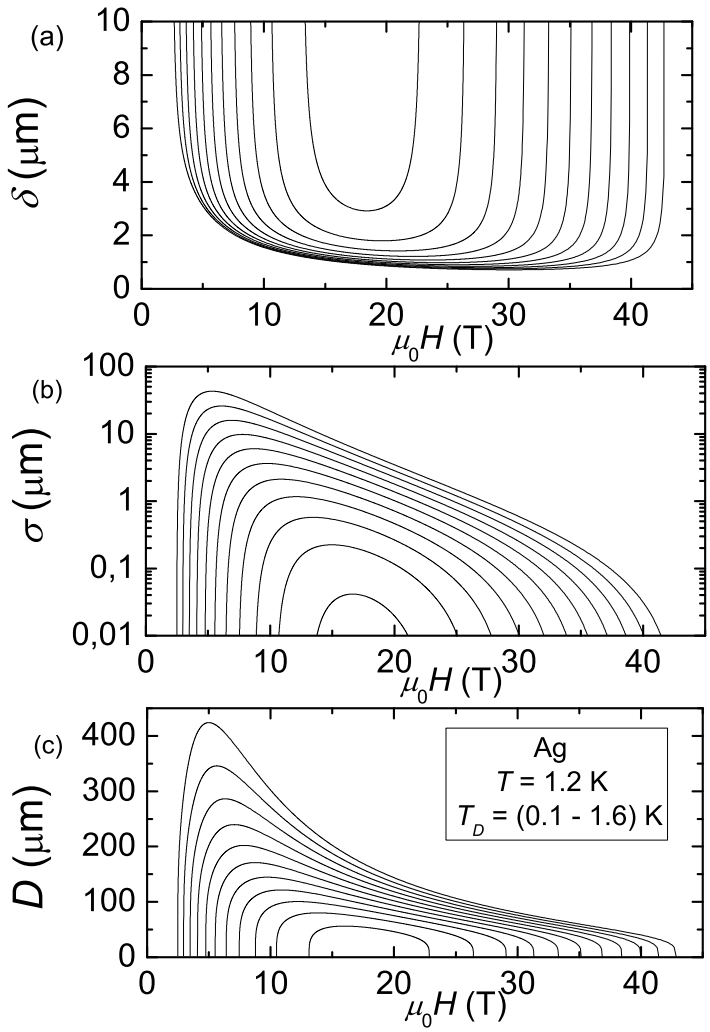

FIG. 3: (a) Magnetic field dependence of DW width $\delta=$ $\delta\left(\mu_{0} H\right)$, (b) specific surface energy $\sigma=\sigma\left(\mu_{0} H\right)$, and (c) period of the domain structure $D=D\left(\mu_{0} H\right)$ at $T=1.2 \mathrm{~K}$ and different Dingle temperatures $T_{D}$. The Dingle temperature increases in steps of $0.15 \mathrm{~K}$ starting from $0.1 \mathrm{~K}$ (from bottom to top in (a) and from top to bottom in (b) and (c)).

Eq. (6) of the CD phase:

$$
\delta=\frac{2 r_{c}}{\alpha^{1 / 2}}, \sigma=5.7 r_{c} \alpha^{3 / 2}, D=5.3\left(r_{c} L\right)^{1 / 2} \alpha^{1 / 4} .
$$

This result is in accordance with the mean-field theory, e. g. near the critical point the length scale of the fluctuations $\sim \delta$ has a power law of divergence with the critical index $\nu=1 / 2$ and the system has no typical scale length $(\sigma, D \rightarrow 0)$ except of the trivial lower $\left(r_{c}\right)$ and upper macroscopic (the size of the system $L$ ) size scales. It should be noted that Eqs. (7) are valid in the range $\left(r_{c} / L\right)^{2 / 3} \ll \alpha \ll 1$ where the low limit value is evaluated from the condition $\delta \ll D / 2$. For $r_{c} \approx 1 \mu \mathrm{m}$ and $L=1 \mathrm{~mm}$ we obtain $\left(r_{c} / L\right)^{2 / 3} \approx 0.01$. Below this value (in the nearest vicinity of critical point $a=1$ ) the periodic domain structure with well-defined DWs transforms into the modulated domain structure [3] .

Due to the bell-like shape of phase diagrams (see, e. g. [7]), there is one critical temperature $T_{c}$ at a given magnetic field and two critical values of the magnetic field $H_{ \pm}$ $\left(H_{-}<H_{+}\right)$at a given temperature. Another possibility for realization of phase transition is related to the concentration of impurities in the sample, which influence the amplitude of dHvA oscillations through the scatter-
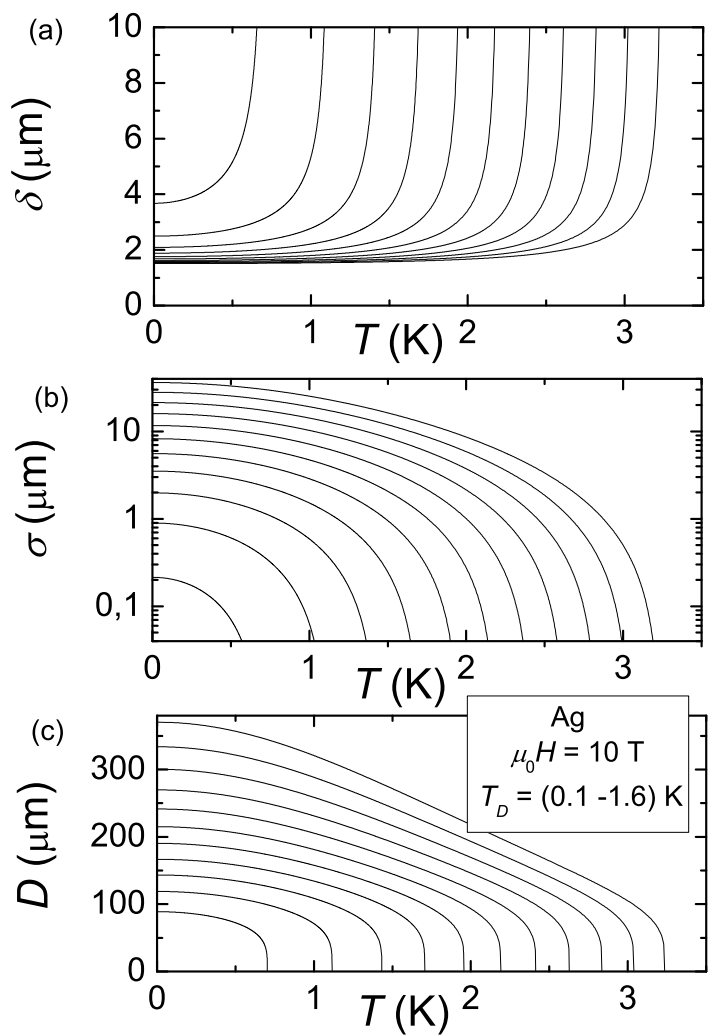

FIG. 4: (a) Temperature dependence of DW width $\delta=\delta(T)$, (b) specific surface energy $\sigma=\sigma(T)$, and (c) period $D=$ $D(T)$ at $\mu_{0} H=10 \mathrm{~T}$ and different Dingle temperatures $T_{D}$. The Dingle temperature increases in steps of $0.15 \mathrm{~K}$ starting from $0.1 \mathrm{~K}$ (from bottom to top in (a) and from top to bottom in (b) and (c)).

ing lifetime $\tau$ of conduction electrons. In the vicinity of critical point $\alpha \rightarrow 0^{+}\left(T \rightarrow T_{c}-0^{+}\right.$, or $H \rightarrow H_{\mp} \pm 0^{+}$, or $T_{D} \rightarrow T_{D, c}-0^{+}$) the temperature and magnetic field dependences of $\alpha$ can be represented as follows

$$
\alpha= \begin{cases}\lambda_{c} L\left(\lambda_{c}\right) t, & t \rightarrow 0^{+}, \\ \lambda_{D, c} t_{D}, & t_{D} \rightarrow 0^{+}, \\ \nu_{\mp} h_{\mp}, & h_{\mp} \rightarrow 0^{+},\end{cases}
$$

where $L(x)=\operatorname{coth} x-1 / x$ is Langevin function and $\pm \nu_{\mp}=-1.5+\lambda_{\mp} L\left(\lambda_{\mp}\right)+\lambda_{\mp}^{D}$. Here, $\lambda_{c}=\lambda\left(\mu_{0} H, T_{c}\right)$, $\lambda_{D, c}=\lambda\left(\mu_{0} H, T_{D, c}\right), \lambda_{\mp}=\lambda\left(\mu_{0} H_{\mp}, T\right)$ and $\lambda_{\mp}^{D}=$ $\lambda\left(\mu_{0} H_{\text {干 }}, T_{D}\right)$. In Eq. (8) $t=1-T / T_{c}, t_{D}=1-T_{D} / T_{D, c}$ and $h_{\mp}= \pm\left(H / H_{\mp}-1\right)$ are small increments of temperature, Dingle temperature and magnetic field for corresponding critical values $T_{c}, T_{D, c}$ and $H_{\mp}$. Substituting Eq. (8) into Eq. (7), one can calculate the temperature and magnetic field dependence of $\delta, \sigma$ and $D$ close to the critical point. In particular, if the phase transition is driven by temperature (at fixed values of $\mu_{0} H$ and $T_{D}$ ) we obtain $\delta \sim t^{-1 / 2}, \sigma \sim t^{3 / 2}$ and $D \sim t^{1 / 4}$.

In other limit, $a \rightarrow+\infty$, one can neglect the second term in free energy density Eq. (11). In this case, the solution of Eq. (3) is $y=2 \tan ^{-1} \sinh \left(\zeta / r_{c}\right)$. Thus, we arrive 
at the following asymptotic behavior of the diamagnetic length scales [21]

$$
\delta \approx 2 r_{c}, \sigma \approx 8 a r_{c}, D \approx 4.89\left(r_{c} L\right)^{1 / 2} a^{1 / 2} .
$$

In the case of $a-1 \gtrsim 1$ one can use the effective free energy density of interacting electrons

$$
G=-\frac{1}{2} K \sin ^{2} \Theta+\frac{1}{2} A\left(\partial_{\zeta} \Theta\right)^{2},
$$

where $\Theta=\pi y / 2 y_{0}$ (see [3] , for details). Parameters $K$ and $A$ are defined as

$$
K=4 a \sin ^{2} \frac{y_{0}}{2}\left(1-a \cos ^{2} \frac{y_{0}}{2}\right), A=a\left(2 r_{c} y_{0} / \pi\right)^{2} .
$$

The first term in Eq. (10) is analogous to the easy-axis crystallographic anisotropy, while the second one corresponds to the exchange interaction in the physics of spin magnetism. It confirms the close analogy between easy-axis anisotropy ferromagnetic sample and the system exhibiting diamagnetic instability. The structure of DW is well known, it is described by the equation $y=\left(2 y_{0} / \pi\right) \tan ^{-1} \sinh (\zeta / \sqrt{A / K})$. In this case, instead of Eqs. (4)-(6) we obtain the following equations for the DW width $\delta$, specific surface energy $\sigma$ and period $D$

$$
\delta=\frac{4 a}{\pi \psi} r_{c}, \sigma=\frac{4}{\pi} y_{0}^{2} \psi r_{c}, D=\frac{2^{5 / 2} \pi\left(\psi r_{c} L\right)^{1 / 2}}{[7 \zeta(3)]^{1 / 2}},
$$

where $\psi=\left[\sec ^{2}\left(y_{0} / 2\right)-a\right]^{1 / 2}$ is a function of $a$. We note that the form of effective energy density Eq. (10) captures the essence of exact calculations in terms of free energy density Eq. (11) even for the regime $0 \leq \alpha \lesssim 1$. Thus, in the most unfavorable case $\alpha \rightarrow\left(r_{c} / L\right)^{2 / 3} \ll 1$ when the expected deviation between the results based on Eqs. (1) and (10) is maximal, the use of effective energy density Eq. (10) gives the same critical behavior Eq. (7) with slightly different numerical factor $\sim 0.01$.

It follows from Eqs. (12) that a monotonic dependence of the characteristic diamagnetic lengths $\delta, \sigma$ and $D$ on the applied magnetic field due to dependence of $r_{c} \sim\left(\mu_{0} H\right)^{-1}$ is mediated by strong non-monotonic dependence on the magnetic field, temperature and purity of a sample through the differential magnetic susceptibility $a=a\left(\mu_{0} H, T, T_{D}\right)$ Eq. (2). Both quantities, $r_{c}$ and $a$, can be evaluated directly in experiments on observation of Condon instability. Fig. [1 shows the diamagnetic length scales $\delta, \sigma$ and $D$ versus differential magnetic susceptibility $a$ Eq. (2) under various magnetic fields $\mu_{0} H$ in the range 2.5-30 $\mathrm{T}$ relevant for appearance of the $\mathrm{CD}$ phase in silver [13]. At values of $a \in[2,5]$ typical for experiment arrangement, the length scales of the CD phase in plate-like sample of silver are: $\delta \sim 1 \mu \mathrm{m}, \sigma \sim 10 \mu \mathrm{m}$ and $D \sim 100 \mu \mathrm{m}$. Under the conditions of experiment on observation of CD structure by NMR measurement 2] $\left(\mu_{0} H=9 \mathrm{~T}, T=1.4 \mathrm{~K}\right.$ and $\left.T_{D}=0.8 \mathrm{~K}\right)$ the theory gives $a=2.6 \mathrm{Eq}$. (2) in accordance with the value calculated from the splitting of the NMR signal (see, e. g. [1]).

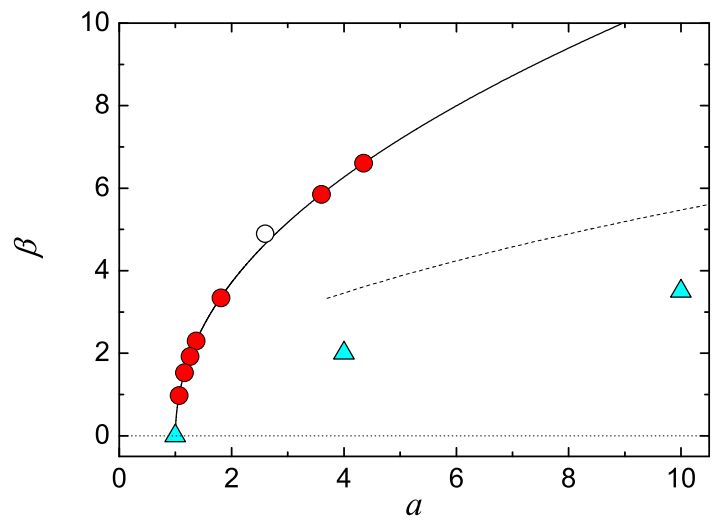

FIG. 5: (color online). Parameter $\beta=D / \sqrt{\delta L}$ Eq. (13) is plotted as a function of differential magnetic susceptibility $a$ in LKS approximation (solid line). The dashed line shows the function $\beta=1.73 a^{1 / 2}$ evaluated in simplified dimensional treatment by Shoenberg [1]. The triangles are Condon's calculation of $\beta$ [22]. Open circle corresponds to data [2], close circle are calculated from the data on measurement of temperature dependence of the magnetic induction splitting [5], as explained in the text.

It follows from Eq. (12) that $\delta \approx 1.5 \mu \mathrm{m}, \sigma \approx 6 \mu \mathrm{m}$ and $D \approx 200 \mu \mathrm{m}$. Similar, in other experiment arrangement, e. g. $\mu_{0} H=10 \mathrm{~T}, T=1.3 \mathrm{~K}$ and $T_{D}=0.2 \mathrm{~K}[5]$, one can calculate $a=4.35$. which gives $\delta \approx 1.3 \mu \mathrm{m}, \sigma \approx 15$ $\mu \mathrm{m}$ and $D \approx 263 \mu \mathrm{m}$.

The results of numerical calculation of the temperature and magnetic field dependences of the diamagnetic lengths $\delta, \sigma$ and $D$ Eq. (12) are illustrated in Fig. 2,4. In calculation of the period $D$ the value of $L=1 \mathrm{~mm}$ is used. Fig. 2 2 shows the magnetic field dependences of the characteristic lengths of the CD phase at constant Dingle temperature $T_{D}=0.1 \mathrm{~K}$ and different temperatures $T$. A family of curves demonstrates the existence of two critical values of the magnetic field in accordance with the phase diagrams [7]. The functions $\sigma=\sigma\left(\mu_{0} H\right)$ and $D\left(\mu_{0} H\right)$ show the existence of maximums which correspond to the maximum of differential magnetic susceptibility $a=a\left(\mu_{0} H\right)$ with a slight shift into the low-field range due to the magnetic field dependence of cyclotron radius $r_{c} \sim\left(\mu_{0} H\right)^{-1}$. The growth of temperature results in the decrease of the interval of values of the magnetic field where the CD phase exists till it collapses around the value of $\mu_{0} H \approx 25$ T. In Fig. 3, the length characteristics of the CD phase are plotted as a function of the magnetic field at fixed temperature $T=1.2 \mathrm{~K}$ and different Dingle temperatures $T_{D}$. The increase in $T_{D}$ due to impurity scattering leads to the reduction of the amplitude of dHvA oscillations similar to the temperature effect, but the impurity effect is more pronounced implying the necessity of using extremely pure samples in studies of Condon instability. The temperature dependences of the length characteristics at a fixed value of the magnetic field $\mu_{0} H=10 \mathrm{~T}$ and different Dingle temperatures are illustrated in Fig. 4. With the increase in the tempera- 

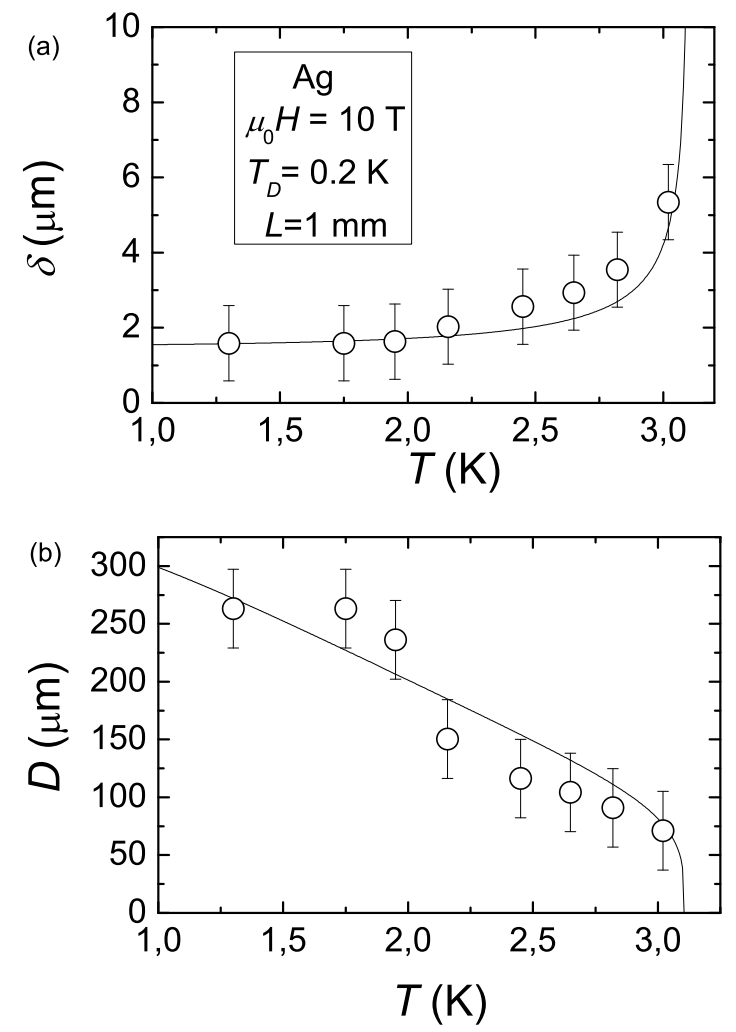

FIG. 6: (color online). Temperature dependence of the DW width $\delta$ (a) and period of the domain structure $D$ (b) at the conditions of the experiment [5]. The solid lines correspond to the theory, the circles are calculated from the temperature dependence of the measured jump of magnetic induction at the interface boundaries [5].

ture, the system approaches the point of phase transition $\left(a \rightarrow 1+0^{+}\right)$where $\delta, \sigma$ and $D$ show critical behavior in a accordance with Eqs. (7) and (8).

It is convenient to introduce a parameter $\beta=$ $D /(\delta L)^{1 / 2}$ independent of the width of the plate $L$ and commonly used in studies of domain structures. It follows from Eq. (12) that $\beta=\beta(a)$ is defined entirely by the properties of correlated electron gas through differential magnetic susceptibility $a$ Eq. (2)

$$
\beta=\frac{(2 \pi)^{3 / 2}}{[7 \zeta(3)]^{1 / 2}} \frac{\psi(a)}{a^{1 / 2}} .
$$

In Fig. [5 parameter $\beta$ Eq. (13) is plotted as a function of $a$ in LKS approximation together with the corresponding estimates due to Shoenberg [1] and Condon [22]. Fig. [5 illustrates essential discrepancy between the theoretical results. In analysis of the domain structure by Shoenberg [1], there are two assumptions. The first assumption is related to the amplitude of dHvA oscillations, e. g. the only case of extremely large values of $a \rightarrow \infty$ when $y_{0}=\pi$ was considered. The second assumption involves the energy of interface boundary $\sigma$ and plays a crucial role in analyzing the period of the domain structure. This energy was evaluated roughly in the order of value as $\sigma \sim a \delta$. It gives the correct asymptotic behavior $D \sim a^{1 / 2}$, but the important numerical factor is missing (see, Eq. (9)). As a result, the minimization of the total energy leads to parameter $\beta=1.73 a^{1 / 2}$. Undoubtedly, the coefficient in the expression remains under question which was also marked by Shoenberg [1] who supposed to use Condon's results instead. Unfortunately, we cannot discuss Condon's estimations 22] which are represented in [1] without prove. As we see below, Condon's result is in contradiction with experiment [5]. In order to calculate a quantity such as the surface energy of the DW Eq. (5), it is necessary to have an expression for the DW structure (3). The calculations in LKS approximation allow us to obtain a correct expression for $\beta$ offering the missing numerical factors for $\sigma$ and $\beta$. In particular, the use of Eq. (9) which is true in a limit $a \rightarrow \infty$ results in $\sigma=4 \delta a^{1 / 2}$ and $\beta=3.45 a^{1 / 2}$.

The confirmation of the validity of our studies comes from the analysis of the data obtained by the Hall probe technique [5]. A set of micro Hall probes was used for detection of local induction at the surface of the pure silver single crystal $2.4 \times 1.6 \times 1.0 \mathrm{~mm}^{3}\left(T_{D}=0.2 \mathrm{~K}\right)$ in the magnetic field up to $10 \mathrm{~T}$ and temperature interval $T=1.3-3 \mathrm{~K}$. The detection of the inhomogeneous induction was attributed to the presence of the CD structure with the period evaluated as being certainly larger than the distance of $\approx 150 \mu \mathrm{m}$ between the edge probes (under limit for period). This result is in contradiction with the proposed in [5] expression $D \approx(L \delta)^{1 / 2}(\beta=1)$ that gives much lower value for $D \approx 30 \mu \mathrm{m}$. Under the conditions of experiment, $a=4.35$ Eq. (2),$r_{c}=0.7 \mu \mathrm{m}$. Thus, according to Shoenberg [1], $\beta=1.73 a^{1 / 2} \approx 3.61$ and $D \approx$ $140 \mu \mathrm{m}$ which is close to, but still less than experimentally determined under limit $150 \mu \mathrm{m}$. The estimates due to Condon results in the lower value of $D \approx 77 \mu \mathrm{m}(\beta \approx$ $2[22])$. On the other hand, calculations in the framework of the presented theory give the same value of $\delta \approx 2 r_{c} \approx$ $1.4 \mu \mathrm{m}$, but the larger value of $D=263 \mu \mathrm{m}$ which is more reasonable because it is above the detected lower limit.

In the case of $\alpha \gtrsim 1$ which is typical for the experiment arrangement on investigation of the CD phase, the change of the magnetic field within the period of dHvA oscillations does not affect the average jump of magnetic induction at the interface boundaries of domain patterns, $\delta B=2 y_{0} / k$ [1], defined by the local magnetization at the center of the $\mathrm{dHvA}$ period, $y_{0}=y_{0}(a)$. It offers a way to calculate the expected values of diamagnetic length scales by means of measurement of the value of $\delta B$. The value of differential magnetic susceptibility $a$ deduced from the measured jump of magnetic induction can be used for evaluation of parameter $\beta$ Eq. (13) and diamagnetic length scales Eq. (12). The results of numerical calculation of $\beta$ from the measurements of temperature dependence of the magnetic field distribution [5] are shown in Fig. 5 Fig. 6illustrates the temperature dependence of the DW width $\delta$ and period of the domain structure $D$ under the conditions of the experiment [5] 
There is a good agreement between the theory and the data.

\section{CONCLUSIONS}

Characteristic diamagnetic length scales of the Condon domain phase in normal metals under quantizing magnetic field and low temperature are studied theoretically in LKS formalism. The results of calculation show that temperature, magnetic field and purity of the sample affect greatly the width of domains and the specific surface energy of DWs, but have little influence on the width of interface boundaries if the system is far enough from the critical point. Well inside the diamagnetic phase, the DW width falls into interval $\sim 1-2 \mu \mathrm{m}$ which is of the same order of value as width of the interface boundary between domains in low-anisotropy magnetic materials such as permalloy thin magnetic films. For a plate-like sample of silver with thickness $\sim 1 \mathrm{~mm}$ a typical value of the period of the diamagnetic domain structure is $\sim 0.1$ $\mathrm{mm}$ which is in two orders of value higher than the average period of ferromagnetic domains for the sample of iron of the same shape.

Approaching at the critical point when $\left(r_{c} / L\right)^{2 / 3} \ll$ $\alpha \ll 1$, the DW width being a characteristic of the range of correlations (coherence length) diverges $\delta \sim \alpha^{-1 / 2}$ with the critical index $\nu=1 / 2$ in accordance with meanfield theory, while the period of the domain structure goes to zero, e. g. the domain structure becomes more dense. The effect of "shrinking" of the domain structure is a result of essential decrease in positive energy of the interface boundary, $\sigma$, which scales as $\sim y_{0}^{3} \sim \alpha^{3 / 2}$. The energy of long-range interaction, $G_{d d}$, scales as $\sim y_{0}^{2}$. Thus, $D \sim \sigma^{1 / 2} / y_{0} \sim \alpha^{1 / 4}$ (see, Eq. (7)). We show that diamagnetic length scales can be calculated from the data on measurement of the jump of magnetic induction at the interface boundaries of domain patterns.

Theoretical results are in agreement with available experimental data. We hope that our studies will stimulate further experimental investigation of diamagnetic length scales in normal metals at the conditions of strong dHvA effect.

\section{Acknowledgments}

We are indebted to V. Egorov, I. Sheikin and D. Golosov for fruitful discussions.
[1] D. Shoenberg, Magnetic Oscillations in Metals (Cambridge University Press, Cambridge, England, 1984).

[2] J. H. Condon and R. E. Walstedt, Phys. Rev. Lett. 21 (1968) 612.

[3] N. Logoboy and W. Joss, Physica B 403 (2008) 3464.

[4] N. Logoboy and W. Joss, Solid State Comm. 146 (2008) 39.

[5] R. B. G. Kramer, V. S. Egorov, V. A. Gasparov, A. G. M. Jansen, and W. Joss, Phys. Rev. Lett. 95 (2005) 267209.

[6] N. Logoboy and W. Joss, Solid State Comm. 139 (2006) 191.

[7] A. Gordon, N. Logoboy and W. Joss. Phys.Rev. B 19 (2004) 174417.

[8] R. B. G. Kramer, V. S. Egorov, A. G. M. Jansen, and W. Joss, Phys. Rev. Lett. 95 (2005) 187204.

[9] N. Logoboy, V. Egorov, and W. Joss, Solid State Comm. 137 (2006) 570.

[10] V. I. Bozhko and E. P. Volskii, Pisma Zh. Eksp. Teor. Fiz. 26 (1977) 337 (Sov. Phys. JETP Lett. 26 (1977) 335).

[11] G. Solt, C. Baines, V. S. Egorov, D. Herlach, E. Krasnoperov and U. Zimmermann, Phys. Rev. Lett. 76, (1996) 2575.

[12] G. Solt, C. Baines, V. S. Egorov, D. Herlach, and U. Zimmermann, Phys. Rev. 59, (1999) 6834.

[13] R. B. G. Kramer, V. S. Egorov, V. A. Gasparov, A. G. M. Jansen, and W. Joss, J. of Phys.: Conf. Series 51 (2006) 299.

[14] M. I. Tsindlekht, N. Logoboy, V. S. Egorov,
R. B. G. Kramer, A. G. M. Jansen, and W. Joss, Phys. of Low Temp. 32 (2006) 1129.

[15] N. Logoboy and W. Joss, Solid State Comm. 139 (2006) 191.

[16] I. A. Privorotskii, Zh. Eksp. Teor. Fiz. 52 (1967) 1755 [Sov. Phys.-JETP 25 (1967) 1755]; I. A. Privorotskii and M.Ya. Azbel, ibid. 56 (1969) 388 [29 (1969) 214]; ZhETF Pis. Red. 5 (1967) 280 [JETP Lett. 5 (1967) 228]; I. Privorotskii, Thermodynamic Theory of Domain Structure (Israel University Press, New York: Wiley and Jerusalem, 1976).

[17] R. C. O'Handley, Modern Magnetic Materials: Principles and Applications (Springer-Verlag, Heidelberg, 2002).

[18] B. Binz, H. B. Braun, T. M. Rice, and M. Sigrist, Phys. Rev. Lett. 96 (2006) 196406.

[19] C. Kittel, Phys. Rev. 70 (1946) 965.

[20] A. Hubert and R. Schäfer, Magnetic Domains - the Analysis of Magnetic Microstructures (A Wiley-Interscience Publication, John Willey and Sons, Inc., 2002).

[21] By direct calculation [16] one can show that the next nonzero term in gradient expantion of free energy Eq. (1) is $-(5 / 48) \operatorname{ar}_{c}^{4}\left(\partial_{\zeta \zeta}^{2}\right)^{2}$. For $a \rightarrow+\infty\left(\delta \rightarrow 2 r_{c}\right)$, the additional term is a factor 10 to 100 smaller than the lowest gradient term and can be taken into account by itteration resulting in a change of DW width withing $\approx 10 \%$. For intermidiate values of $a\left(\delta>>d_{c}=2 r_{c}\right)$ the error should be smaller due to the presence of prefactor $\left(d_{c} / \delta\right)^{4}<<1$.

[22] J. H. Condon, unpublished (see, [1], p.274). 